\title{
The Research and Realization of A Localization Algorithm in WSN Based on Multidimensional Scaling
}

\author{
Li Xiang, Qianzhi Hong, Liuxiao Hui \\ College of Communication Engineering, Jilin University, Changchun,China \\ e-mail:Ix448027120@163.com,dr.qzh@163.com, 1066058585@qq.com
}

Keywords: multidimensional scaling; wireless sensor networks; sensor localization; TDOA

\begin{abstract}
A new locating algorithm NMDS-TDOA is proposed, which attempts to improve the performance of node localization in wireless sensor networks. As the ranks of the nodes based on TDOA should be monotonic with their ranks based on true distance, we can combine TDOA and MDS-MAP. As a result, positioning error will be reduced. The process of the computation about turning nodes' relative coordinates into absolute coordinates is introduced explicitly. NMDS-TDOA realizes both less complexity and relatively accurate positioning only based on few anchors, even in the situation of lacking information on the distance between nodes.
\end{abstract}

\section{Introduction}

In recent years, wireless sensor technology develops rapidly. It is widely used in areas such as military, transportation, environment and industrial production, to finish the measurement of many physical quantities such as temeperature, humidity, pressure and speed. For most of the measured quantities, people not only need to master the accurate measurement values, also need to know the area or location of the measured quantities. However, sensor nodes of WSN are generally batch deployment, such as aerial seeding, and the structure and function of each node are exactly the same. So we can't separate each node identification. Therefore, to obtain the location of measured values, the sensor nodes must be able to clear their own locations. The location information of the sensor nodes can also help to improve the quality of WSN routing efficiency, coveraging quality, realizes the network load balancing and since topology configuration,etc. How to get the node location information problem called positioning problem of sensor nodes. Now this problem has become one of the hot issues in the field of WSN. MDS-MAP ${ }^{[1-2]}$ algorithm was proposed by Shang, then many more improved algorithms ${ }^{[3-4]}$ were proposed.

In view of the sensor node positioning problem of WSN, this paper proposes a new node localizantion algorithm, and gives the specific algorithm of how to turn nodes' relative coordinates into absolute coordinates. TDOA signal is used in this proposed method to locate, so that the caculation error and computations brought by converting signal strengh values into distance values in previous localizationg algorithms will be reduced. NMDS-TDOA is proved a good positioning result and certain robustness through the analysis of combination of the theory and practice. All algrithms introduced below are discussed in 2-dimensional space.

\section{Multidimensional Scaling(MDS)}

The multidimensional scaling(MDS), a technique usually used for the analysis of exploratory data analysis or information visualization, was first used in data analysis of psychometrics. Now MDS is widely used in many fields as a general data analysis technology. MDS uses diversity between entities to compose relative coordinate in multi-dimensional space. The more similar two entities, the shorter distance between the points which the entities correspond to in the space.

The main advantage in using MDS for location estimation is that it can generates accurate location estimation even based on few anchor nodes. There are numerous varieties of MDS. We focus on classical MDS and nonmetric MDS. 


\section{Classical Multidimensional Scaling}

In classical multidimensional scaling, we assume $\mathrm{p}_{i j}$ represents diversity between entity $\mathrm{i}$ and entity $\mathrm{j}$. The diversity matrix $\mathrm{P}$ is composed with $p_{i j}$ which is the euclidcan distance between nodes. The basic idea is to reconsitute the coordinates which the nodes coordinate to in multi-dimensional space by using matrix $\mathrm{P}$, in order to minimize stress coefficient.

$$
\text { STRESS }=\sum\left(f\left(p_{i j}\right)-d_{i j}\right)^{2}
$$

If all pairwise distances of sensors in $\mathrm{P}$ are collected, we can use classical multidimensional scaling algorithm to estimate the locations of sensors:

1) Compute the matrix of squared distance $P^{2}$;

2) Compute the matrix $J$ with $J=E-n^{-1} \square$, where $\mathrm{E}$ is identity matrix,and $\mathrm{I}$ is a full one square matrix;

3) Apply double centering to this matrix with $B=-\frac{1}{2} J\left[P^{(2)} \square\right.$.Double centred means every element in the matrix minus the row mean value and the column mean value,then plus the mean value of the matrix, finally multiply by $-1 / 2$;

4) $\mathrm{B}$ is decomposed by singular value decomposition, so that we can get eigenvalues $\lambda_{1}, \lambda_{2}, \cdots \lambda_{m}$ which composed diagonal matrix $\Lambda$, and eigenvectors $e_{1}, e_{2}, \cdots e_{m}$ which composed matrix $\mathrm{V}$. The coordinate matrix of classical scaling is $X=V \square \Lambda^{1 / 2}$.

\section{Nonmetric Multidimensional Scaling}

The relation between diversity and distance is not requested as strict as classical multidimensional scaling in nonmetric multidimensional scaling. It's only asked to meet the monotonic relation, not quantitatively presented. To entity $\mathrm{i}, \mathrm{j}, \mathrm{u}, \mathrm{v}$, if there is $\mathrm{p}_{i j}<p_{u v}$, then $d_{i j}(X)<d_{u v}(X)$.

It's a repetitive iteration process to reconsitute the coordinates and distance based on diversity between the entities in nonmetric MDS ${ }^{[1-3]}$ :

1) Initialization phase. Calculate Euclidean distance $d_{i j}^{0}$ between entities by random initial coordinate $X^{0}$. And each initial coordinate must be different from another;

2) A median called disparities is generated which is expressed by $\hat{d}_{i j}$. $\hat{d}_{i j}^{0}$ and $p_{i j}$ must meet monotonic relation, which means to $\mathrm{i}, \mathrm{j}, \mathrm{u}, \mathrm{v}$, if there is $p_{i j}<p_{u v}$, there is $\hat{d}_{i j}^{0} \leq \hat{d}_{u v}^{0}$. We could use PAV(pool-adjacent violators) algorithm to get $\hat{d}_{i j}^{0}$ :

a) The entities are sorted from small to large based on $p_{i j}$;

b) Compare distance between entities $d_{i j}^{0}$ until $d_{i j}^{0}$ does not meet monotonic relation;

c) Raplace $\hat{d}_{i j}^{0}$ with the average value of $d_{i j}^{0}$;

d) Deal with the next $p_{i j}$, then repeat b and c until all $d_{i j}^{0}$ are done and get all $\hat{\mathrm{d}}_{i j}^{0}$;

3) Change $X^{0}$ to $X^{1}$ and calculate Euclidean distance $d_{i j}^{1}$.

Caculate $\hat{\mathrm{d}}_{i j}^{\mathrm{k}}$ and $\mathrm{d}_{i j}^{k}$ in the loop interation composed by the above 3 steps until STRESS1 satisfies some certain request.

$$
\text { STRESS } 1=\sqrt{\sum_{i j, i \neq j}\left(\hat{\mathrm{d}}_{i j}-d_{i j}\right)^{2} / \sum_{i j, i \neq j} d_{i j}^{2}}
$$




\section{The NMDS-TDOA Algorithm}

NMDS-TDOA proposed in this paper meets the request of nonmetric MDS, which is the larger distance value between nodes, the larger TDOA value we get. The detailed process is as follows:

Input: TDOA values between nodes.

Outpt: Absolute coordinate of the nodes in the network.

Step1. Compose $\left[\mathrm{s}_{i j}\right]$ with TDOA values;

Step2. Get $\left[p_{i j}\right]$ by dealing $\left[\mathrm{s}_{i j}\right]$ with Dijkstra algorithm, the shortest path algorithm;

Step3. Deal $\left[p_{i j}\right]$ with nonmetric MDS to get the relative coordinates:

a) Give nodes initial coordinate $\left(x_{i}^{0}, y_{i}^{0}\right)$;

b) Caculate the Euclidean distance $d_{i j}^{k}=\sqrt{\left(x_{i}^{k}-x_{j}^{k}\right)^{2}+\left(y_{i}^{k}-y_{j}^{k}\right)^{2}}$;

c) Deal $\left[p_{i j}\right]$ and $\left[d_{i j}^{k}\right]$ with PAV algorithm to get $\left[\hat{d}_{i j}^{k}\right]$ :

To envity $\mathrm{i}, \mathrm{j}, \mathrm{u}, \mathrm{v}$, if $\mathrm{p}_{i j}<p_{u v}$ and $\mathrm{d}_{i j}^{k}>d_{u v}^{k}, \hat{d}_{i j}^{k}=\hat{d}_{u v}^{k}=\left(d_{i j}^{k}+d_{u v}^{k}\right) / 2$;

If $\mathrm{p}_{i j}<p_{u v}$ and $\mathrm{d}_{i j}^{k}<d_{u v}^{k}, \hat{d}_{i j}^{k}=d_{i j}^{k}, \hat{d}_{u v}^{k}=d_{u v}^{k}$;

d) $\mathrm{k}=\mathrm{k}+1$, caculate the new coordinates $\left(x_{i}^{\mathrm{k}}, y_{i}^{\mathrm{k}}\right)$ :

$$
\begin{aligned}
& x_{i}^{k}=x_{i}^{k-1}+\frac{\alpha}{n-1} \sum_{j \in M, j \neq i}\left(1-\frac{d_{i j}^{k-1}}{d_{i j}^{k-1}}\right)\left(x_{j}^{k-1}-x_{i}^{k-1}\right), \\
& y_{i}^{k}=y_{i}^{k-1}+\frac{\alpha}{n-1} \sum_{j \in M, j \neq i}\left(1-\frac{d_{i j}^{\hat{k}-1}}{d_{i j}^{k-1}}\right)\left(y_{j}^{k-1}-y_{i}^{k-1}\right),
\end{aligned}
$$

$\mathrm{n}$ is the number of the nodes, $\alpha$ is the iteration step, $\alpha=2$;

e) Update the Euclidean distance with the new coordinates;

f) Caculate STRESS1. If $S T R E S S 1<\varepsilon$, end the loop, else back to c. $\varepsilon=10^{-4}$;

Step4. Turn relative coordinates into absolute coordinates based on the coordinates of the anchors:

The alignment usually includes shift, rotation, and reflection of coordinates. $\mathrm{R}=\left[r_{i j}\right]_{2 \times n}=\left(R_{1}, R_{2}, \ldots, R_{n}\right)$ denotes the relative locations of the set of $\mathrm{n}$ sensor nodes. $\mathrm{T}=\left[\mathrm{t}_{i j}\right]_{2 \times n}=\left(T_{1}, T_{2}, \ldots, T_{n}\right)$ denotes the true locations of $\mathrm{n}$ sensor nodes. In following explanation, we assume the node 1, 2, 3 are anchors. A vector $R_{i}$ may be shifted to $R_{i}^{1}$ by $R_{i}^{1}=R_{i}+X$, where $X=R_{i}^{1}-R_{i}$. It may be rotated counterclockwise through an angle $\alpha$ to $\mathrm{R}_{i}^{2}=Q_{1} R_{i}$, where

$Q_{1}=\left[\begin{array}{cc}\cos (\alpha) & -\sin (\alpha) \\ \sin (\alpha) & \cos (\alpha)\end{array}\right]$.

It may also be reflected across a line

$S=\left[\begin{array}{l}\cos (\beta / 2) \\ \sin (\beta / 2)\end{array}\right]$

to $\mathrm{R}_{i}^{3}=Q_{2} R_{i}$, where

$Q_{2}=\left[\begin{array}{cc}\cos (\beta) & \sin (\beta) \\ \sin (\beta) & -\cos (\beta)\end{array}\right]$. 


\section{Test results}

In our experiments, we run NMDS-TDOA on various topologies of networks in Matlab. The nodes are placed both randomly and on a square grid. Location error could be caculated by formula (8).

error $=\frac{\sum_{i=m+1}^{n}\left\|X_{\text {est }}^{i}-X_{\text {real }}^{i}\right\|^{2}}{(n-m) R} \times 100 \%$

\section{Random Placement}

In this set of experiments, 50 nodes are placed randomly in a $10 \times 10$ square.Figure 1 shows an example using a radio range of 3 , which leads to an average connectivity of 11.56 . The green lines represent the connectivity between neighbor nodes. Figure 2 shows the final result of NMDS-TDOA which is transformed based on 3 anchor nodes, denoted by red stars in the network. The red line represent the difference between the estimated position and the true position. The longer the line, the larger the error.The average estimation error in this example is about $14.30 \%$.

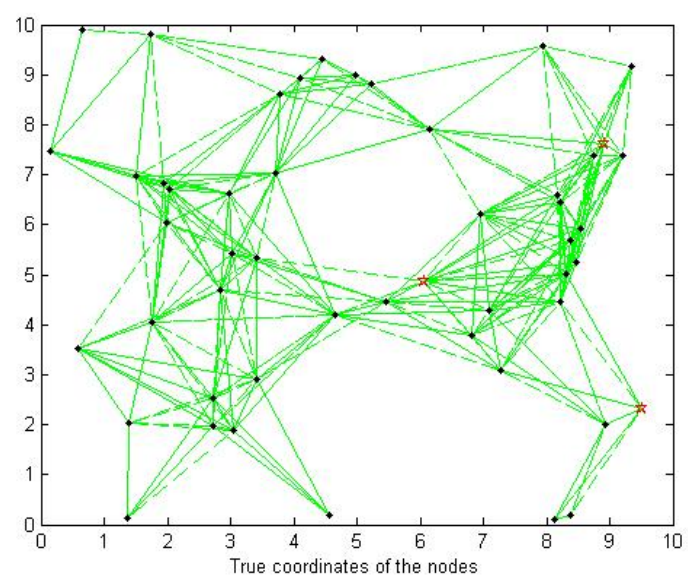

Fig.1 True coordinates of the nodes

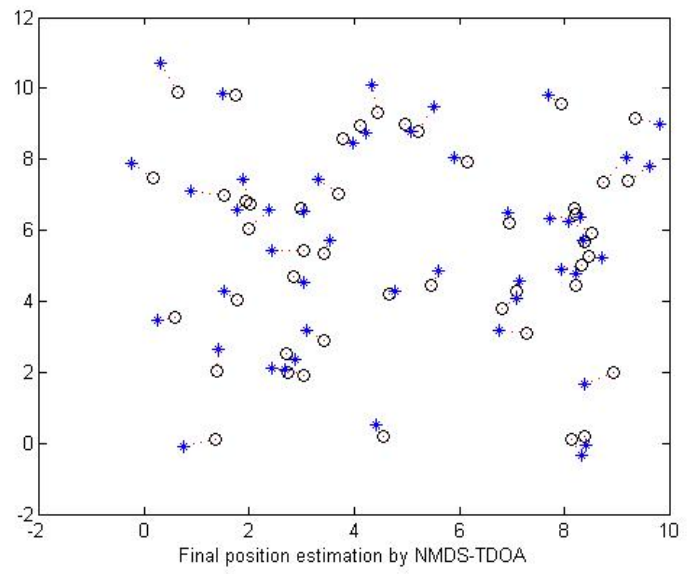

Fig.2 Final position estimation by NMDS-TDOA

\section{Grid Placement}

In this set of experiments, we assume that the sensor nodes are placed as a square.We could add random placement error to these nodes. 49 nodes are placed on a $7 \times 7$ grid. Figure 3, figure 4 and figure 5 show an example of no placement error. The radio range is $\mathrm{R}=3$, which leads to connectivity 17.16. Green lines also represent the connections between neighbors. Figure 3 shows the true coordinates of the nodes. Figure 4 shows the relative coordinates of NMDS-TDOA. Figure 5 shows the final result of NMDS-TDOA. The average estimation error in this example is about $10.23 \%$.

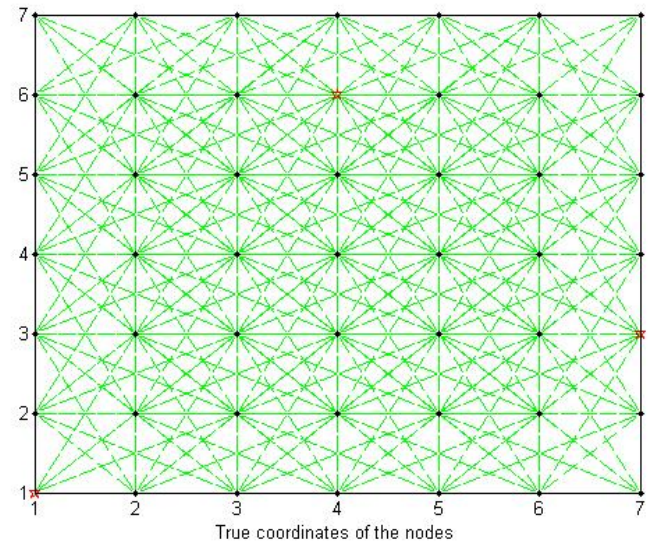

Fig.3 True coordinates of the nodes in grid placement

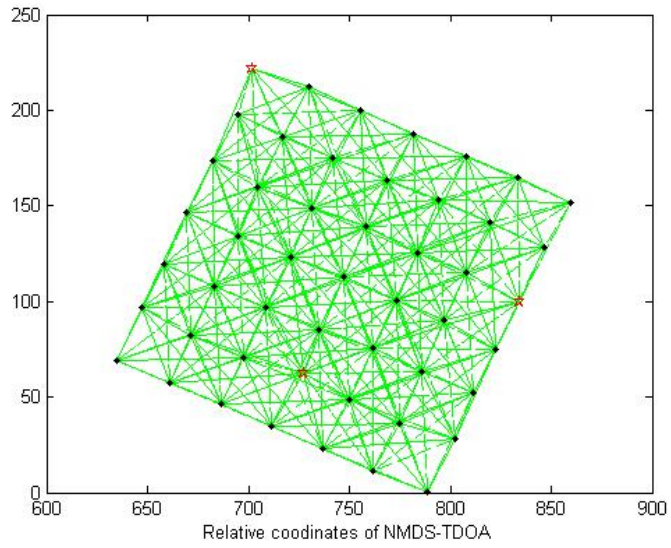

Fig.4 Relative coordinates of NMDS-TDOA 


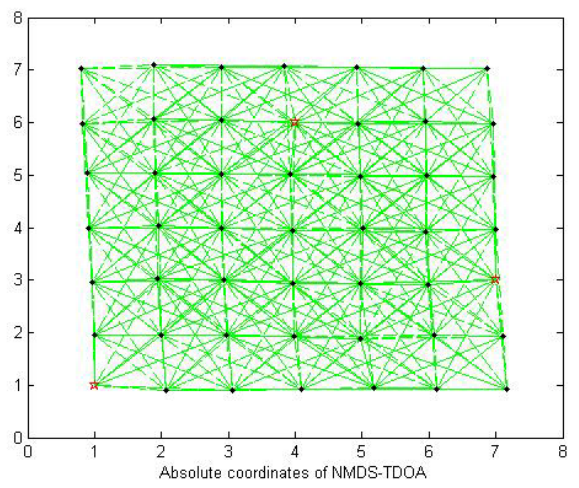

Fig.5 Absolute coordinates of NMDS-TDOA

Figure 6 shows the average performance of NMDS-TDOA as a function of connectivity. And NMDS-TDOA is compared with MDS-MAP ${ }^{[1-3]}$ which only uses connectivity information at the same time. The radio ranges are 2, 2.5, 3, 3.5, 4, 4.5. From the figure, we can see that the errors with 3 anchors are quite good when the connectivity level is 12.2 or greater. The result of grid placement in this figure is got with $5 \%$ placement error.
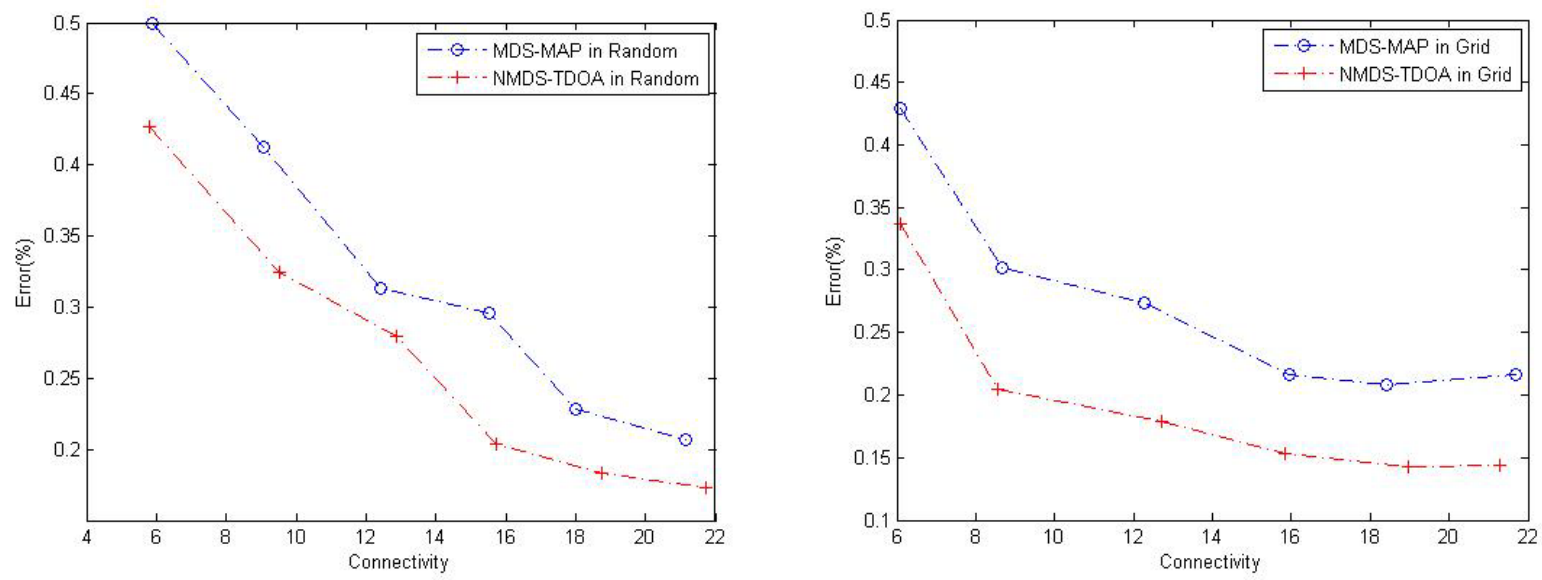

Fig.6 Average localization error as a function of connectivity

\section{Conclusion}

NMDS-TDOA proposed in this paper uses only few anchors to realize a relatively accurate positioning. This algorithm takes full advantage of nonmetric MDS, which only needs signals keep monotonic with distance between the nodes. The caculation error and computations brought by converting signal strengh values into distance values in previous localizationg algorithms will be reduced. It is testified by theory and practice that NMDS-TDOA has a smaller location error.

\section{References}

[1] Yi Shang, Wheeler Ruml, Ying Zhang, et al. Localization from Mere Connectivity in Sensor Networks [C]. In: Proc of the 4th ACM Int' Symp on Mobile Ad Hoc Networking \& Computing. New York: ACM Press, 2003. 201-212.

[2] Yi Shang, Wheeler Ruml, Ying Zhang. Localization from Connectivity in Sensor Networks [J]. IEEE Trans on Parallel and Distributed Systems, 2004, 15(11): 961-973.

[3] Ma Zhen, Liu Yun, Shen Bo. Distributed Location Algorithm for Wireless Sensor Networks MDS-MAP(D) [J]. Journal of Communications, 2008, 29(6):57-62.

[4] Chen Suishen, Lu Jiangang, Lou Xiaochun. Localization Algorithm for Wireless Sensor Networks Based on MDS-MAP and Nonlinear Filtering [J]. Journal of Zhejiang University(Engineering Science), 2012, 46(5): 866-872

[5] Zhang Lu, Fan Wei, Han Shuangxia, et al, Design and Realization of the Distributed Localization Algorithm Based on MDS-MAP for WSNs [J]. Computer \& Digital Engineering, 2013, 41(6): 876-879. 